MATEC Web of Conferences 43, 01004 (2016)

DOI: $10.1051 /$ matecconf/20164301004

(C) Owned by the authors, published by EDP Sciences, 2016

\title{
Preparation and Characterization of Polyurethane Films Using Corn (Zea mays L.) Oil-based Polyol
}

\author{
Francis Louise J. Fajardo, John Ralou M. dela Peña, Juchris D. Bayani and Eduardo R. Magdaluyo, Jr. ${ }^{\text {a }}$ \\ Department of Mining, Metallurgical and Materials Engineering, College of Engineering, University of the Philippines, 1101 Diliman,
}

Quezon City, Philippines

\begin{abstract}
The study focused on the synthesis of polyurethane films utilizing corn (Zea mays L.) oil-based polyol. The activation of hydroxyl sites of the linoleic acid and other fatty acids present in the corn oil was done via epoxidation followed by hydroxylation process. Afterwhich, the activated polyol was reacted with hexamethylene diisocyanate to produce the polyurethane. Variations in the $\mathrm{NCO} / \mathrm{OH}$ ratio and reaction time were carried to assess the effect on the synthesis of the polymer and its properties. Infrared spectral analyses showed that the polyol conversion was successful due to the presence of epoxy binding sites at 1100 to $1200 \mathrm{~cm}^{-1}$ and the hydroxyl groups at 3400 to $3600 \mathrm{~cm}^{-1}$. The formation of polyurethane was confirmed in the infrared spectral analyses. The characteristic bonds of urethane groups were observed at $3330 \mathrm{~cm}^{-1}$ due to $-\mathrm{OH}, 2947 \mathrm{~cm}^{-1}$ associated to symmetric $-\mathrm{CH}$ and $2810 \mathrm{~cm}^{-1}$ due to asymmetric stretching vibration of $-\mathrm{CH}_{2}$. The reaction time held no significant change to the composition of the polymer while an increase in $\mathrm{NCO} / \mathrm{OH}$ ratio entailed rigidity of the film. The $2: 1 \mathrm{NCO} / \mathrm{OH}$ ratio at three hours reaction showed a balance of desirable mechanical properties and processability. Thermal decomposition characteristics of the polyurethane showed three distinct temperatures as the $\mathrm{NCO} / \mathrm{OH}$ loading increases to $3: 1$ ratio. The maximum tensile strength observed was at $0.285 \mathrm{MPa}$ at $0.45 \mathrm{~mm}$ film thickness.
\end{abstract}

\section{Introduction}

Polyurethane is known as a large family of polymer with repeating hard and soft segments connected by means of urethane linkage due to the reaction of diisocyanate and polyol functional groups. This kind of diverse polymer structure yields great property distinction as well as tunable applications. The various uses include insulation, coating, sealant, adhesive, cement additive as well as in tissue engineering and thermal energy storage [1-5]. As the reactants are derived from non-renewable sources, the chemical industry hopes to shift to self-sustaining sources for high batch production at minimal costs [6]. In particular, vegetable oil-based polyol used in polyurethane synthesis offers an environmental and economic advantage that can substitute its petrochemical based counterpart [7].

Particularly for oils, the content of triglycerides or unsaturated fatty acids can be the source of polyol functional group. But as oils are relatively unreactive for polymer synthesis, the functionality must be activated through modification of the double bonds present in the triglycerides. The type of vegetable oil to be activated must be considered, especially looking into the fatty acid concentrations in order to find the appropriate reactive site for polyol conversion as it can affect the polymer properties [8-9]. Looking at fatty chain structures, those with major components such as linoleic acid (C18:2) are

\footnotetext{
${ }^{\mathrm{a}}$ Corresponding author: ed.magdaluyojr@coe.upd.edu.ph
}

known reactive sites through the presence of the double bonds, while those saturated fatty acids such as lauric acid (C12:0) must be reacted through the glyceride ester linkage [10].

In the conversion of vegetable oils to polyols, various processes can be employed in producing the active $-\mathrm{OH}$ site needed for polymerization reaction. Among processes such as ozonolysis, pyrolysis and alkali fusion [11], the frequent procedure in manipulation of the oil is through formation of a reactive epoxidize group that can be treated by ring-opening or hydroxylation and acrylation $[9,12]$.

Locally abundant oils in the Philippines such as corn oil (Zea mays L.) show a potential source as raw material because it is renewable and biodegradable. Thus, the main purpose of this research was to produce a polyurethane film from locally produced corn oil as polyol source. The preparation involved the activation of the corn oil via epoxidation and followed by hydroxylation. The spectral, mechanical and thermal properties of the produced film were characterized and the optimal $\mathrm{NCO} / \mathrm{OH}$ ratio and reaction time for the polymer were also investigated.

\section{Experimental procedures}


The corn oil (Zea mays L.) was activated for polyol conversion via a modified procedure on epoxidation, followed by hydroxylation [13]. A calculated amount of 0.05 mol of corn oil was heated to $90^{\circ} \mathrm{C}$ with $0.3 \mathrm{~mol}$ formic acid in three-necked round bottom flask with reflux set-up at constant stirring. A $1.5 \mathrm{~mol}$ of $30 \%$ hydrogen peroxide was added drop-wise and the reaction was carried out for 6 hours. Upon completion, the solution was allowed to cool at room temperature before extraction of the oil via centrifugation. Following epoxidation, a stoichiometric quantity of water, formic acid, and methanol were mixed in the same set-up utilized earlier. The oil was added to the mixture upon heating at $60^{\circ} \mathrm{C}$. After hydroxylation for 30 minutes, the mixture was left to cool and the oil was extracted again via centrifugation. The obtained polyol was then reacted with hexamethylene diisocyanate (HMDI) in the following $\mathrm{NCO} / \mathrm{OH}$ ratios: $1: 1,2: 1$, and $3: 1$. The volume of the polyol was kept at $5 \mathrm{ml}$ and the reaction was carried out at $90^{\circ} \mathrm{C}$ under constant stirring. Variations in reaction time were 3, 4 and 5 hours. The synthesized polyurethane was casted and cured for 48 hours at room temperature.

Compositional analyses of the as-received, epoxidized, hydroxylated corn oil and synthesized polyurethane were obtained via fourier transform infrared spectroscopy (FTIR). The mechanical properties were determined via stress-strain curves obtained from universal testing machine (UTM) following ASTM D412-06A as modified. Rectangular samples were prepared $(4 \mathrm{~cm} \mathrm{x} 1 \mathrm{~cm})$ at varying thickness and the test was done at strain rate of $5 \mathrm{~mm} / \mathrm{min}$. The thermal properties of the were also investigated using thermogravimetric analyser (TGA) and differential scanning calorimeter (DSC).

\section{Results and discussion}

The reaction from the polyol conversion of corn oil involves the functionalization of the double bonds present in the main component of the vegetable oil, which is generally composed of $70 \%$ linoleic acid [14]. The oxidative hydrogen peroxide and formic acid catalyst are responsible for the formation of an epoxy groups from the double bonds of the different fatty acids [10]. After which, ring-opening via methanol, water and formic acid would produce the activated polyol through the hydroxyl bond formed from the epoxide site. Figure 1 shows the general mechanism of the activation process and the obtained corn oil-based polyurethane film as-casted is shown in Figure 2.<smiles>CCCCC/C=C\C/C=C\CCCCCCCC(=O)O</smiles><smiles>CCCCCC(O)C(CC(O)C(C)CCCCCCCC(=O)O)OC</smiles>

Figure 1. Reaction mechanism for the activation of polyol from linoleic acid via epoxidation and hydroxylation.
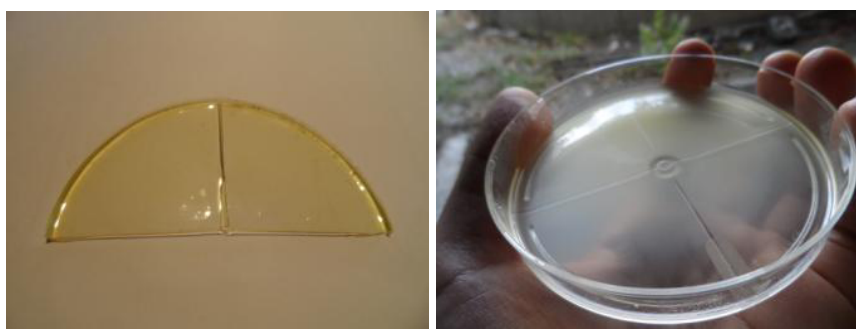

Figure 2. The fabricated corn oil-based polyurethane film.

The unsaturated bonds at $3007 \mathrm{~cm}^{-1}$ based on infrared spectral analyses of the as-received corn oil in Figure 3 started to become less apparent as the conversion of the vegetable oil proceeded from epoxidation to hydroxylation. The binding sites present in the epoxidized and hydroxylated corn oil spectra are the epoxy group at the range of $1100-1200 \mathrm{~cm}^{-1}$ and the hydroxyl (-OH groups) at $3400-3600 \mathrm{~cm}^{-1}$. The absence of the active hydroxyl binding sites in the as-received corn oil makes it difficult for the reaction with diisocyanate to form polyurethane.

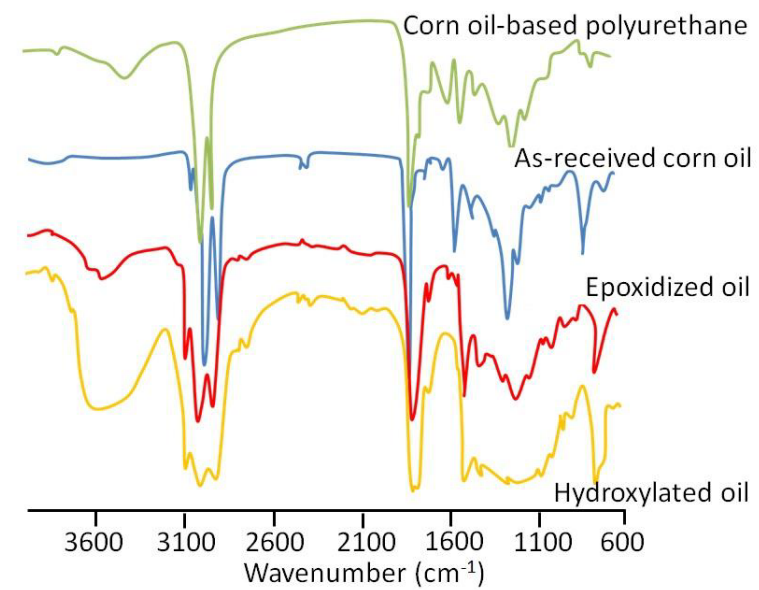

Figure 3. Infrared spectra of corn oil-based polyurethane, asreceived oil as well as the epoxidized and hydroxylated corn oil

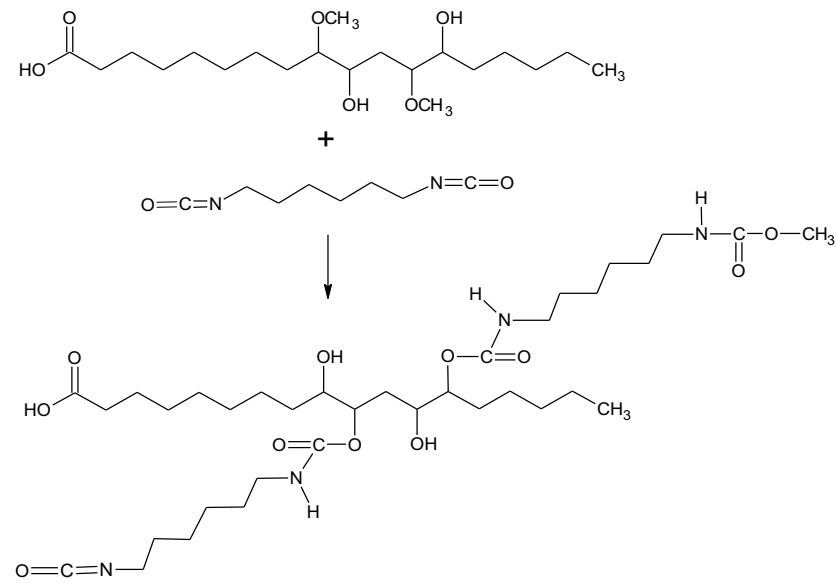

Figure 4. Polymer reaction of hydroxylated corn oil-based polyol and hexamethylene diisocyanate. 
The infrared spectral analyses of the corn oil-based polyurethane showed the characteristic bands of urethane groups at 3330 (-OH groups), 2947 ( $-\mathrm{CH}$ symmetric) and $2810 \mathrm{~cm}^{-1}$ (asymmetric stretching vibrations of $-\mathrm{CH}_{2}$ ). The peaks observed at $1728,1642,1599 \mathrm{~cm}^{-1}$ were

all associated to $-\mathrm{CO}$ bonds and $1529 \mathrm{~cm}^{-1}$ due to $-\mathrm{NH}$ deformation. The reaction mechanism of the hydroxylated corn oil-based polyol and the hexamethylene diisocyanate is shown in Figure 4.

Figure 5 shows the infrared spectra of the obtained corn oil-based polyurethane at varying $\mathrm{NCO} / \mathrm{OH}$ ratio and reaction time. The characteristic bands of the urethane groups were present in all ratios; however, the final viscoelastic property of the as-cast polyurethane film showed significant difference. The corn oil-based polyurethane film with the $1: 1 \mathrm{NCO} / \mathrm{OH}$ ratio resulted to soft film while the sample with $2: 1 \mathrm{NCO} / \mathrm{OH}$ had the right flexibility. The film with $3: 1 \mathrm{NCO} / \mathrm{OH}$ ratio showed brittle characteristic. This property can be attributed to the increase in concentration of the hard segments found in the aliphatic diisocyanate that would increase crosslinking, and ultimately, the toughness of the film [15]. With the consideration of processability, the corn oil-base polyurethane film with $2: 1 \mathrm{NCO} / \mathrm{OH}$ ratio was further tested for mechanical and thermal properties.

There is also no notable changes appeared in the characteristic bands of polyurethane regardless of the reaction time. Visual inspection of the films indicated that an increase in reaction time would make the polymer more viscous. Hence, the three hour reaction time showed less viscosity that will entail easy casting for film fabrication. The infrared spectral analyses of the corn oilbased polyurethane showed the characteristic bands of urethane groups at 3330 ( $-\mathrm{OH}$ groups), $2947(-\mathrm{CH}$ symmetric) and $2810 \mathrm{~cm}^{-1}$ (asymmetric stretching vibrations of $-\mathrm{CH}_{2}$ ). The peaks observed at 1728,1642 , $1599 \mathrm{~cm}^{-1}$ were all associated to $-\mathrm{CO}$ bonds and $1529 \mathrm{~cm}^{-1}$ due to $-\mathrm{NH}$ deformation.

As solvent casting was employed in film formation, varying thickness of the corn oil-based polyurethane films were produced. The behaviour of the polymer exhibits that of a thermoset plastic where elastic deformation is present up until the point of fracture, based on the stress-strain curve as shown in Figure 6. Similar behaviour was also observed in a study that employed fatty acids in polyurethane synthesis [16].

Observing the various thicknesses of as-casted corn oil-based polyurethane films at 2:1 $\mathrm{NCO} / \mathrm{OH}$, those with low or high value of thickness did not perform well in the tensile stress test with failures before the $0.1 \mathrm{~mm} / \mathrm{mm}$. Polyurethane films with good mechanical properties have thickness at the range of 0.25 to $0.40 \mathrm{~mm}$, particularly the $0.45 \mathrm{~mm}$ thickness showed the highest resilience and the tensile strength at $0.285 \mathrm{MPa}$.

Figure $7 \mathrm{a}$ shows the decomposition characteristics of the corn oil-based polyurethane at different $\mathrm{NCO} / \mathrm{OH}$ loading based on the thermo gravimetric analyses under nitrogen environment. Based on the derivative weight percent (broken lines) profile for the 3:1 NCO/OH ratio, there are three distinct decomposition temperatures at around 350,415 and $456^{\circ} \mathrm{C}$. The $1^{\text {st }}$ decomposition temperature is not visible in the polyurethane sample with
$1: 1 \mathrm{NCO} / \mathrm{OH}$ loading and just an inflection as seen in the sample having 2:1 NCO/OH ratio. The observed increase of the intensity at $456^{\circ} \mathrm{C}$ and the corresponding decrease in the $2^{\text {nd }}$ decomposition temperature is attributed to the increase amount of hard segment due to higher isocyanate component.
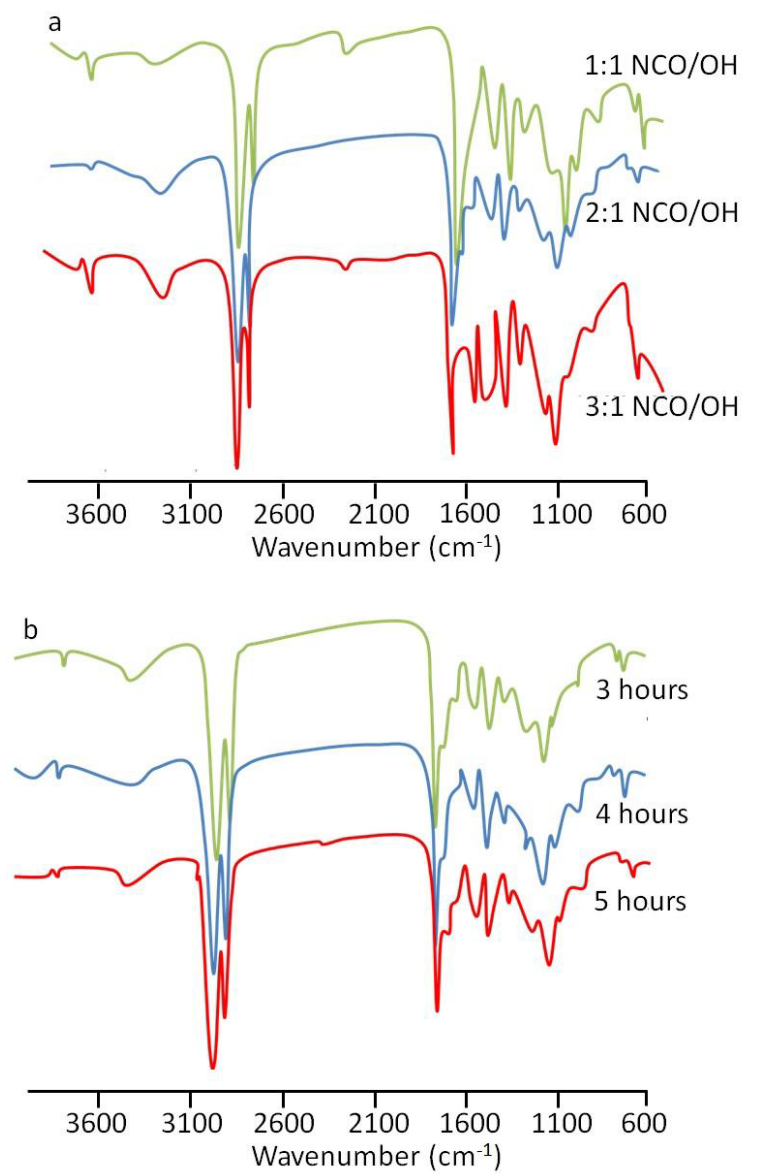

Figure 5. Infrared spectra of corn oil-based polyurethane with varying $\mathrm{NCO} / \mathrm{OH}$ ratio (a) and varying reaction time (b)

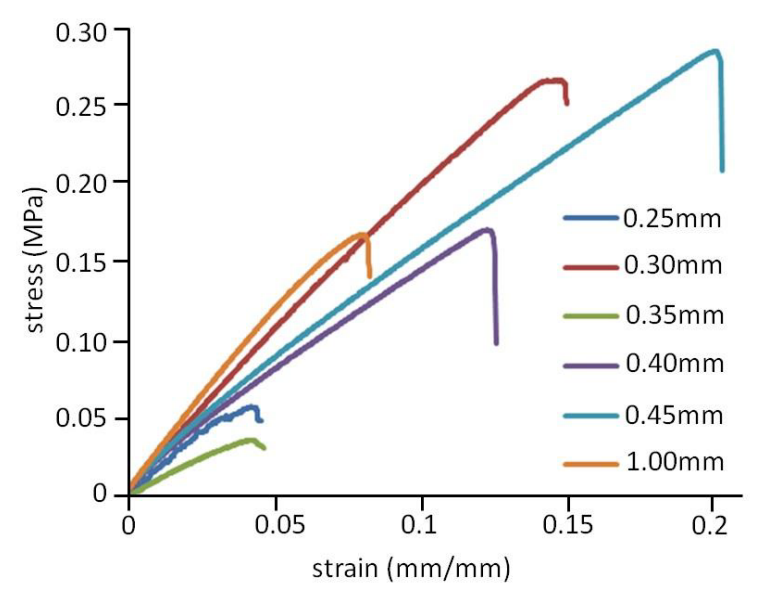

Figure 6. Stress-strain curve of corn oil-based polyurethane with $1: 2 \mathrm{NCO} / \mathrm{OH}$ ratio at $3 \mathrm{hrs}$ reaction time. 

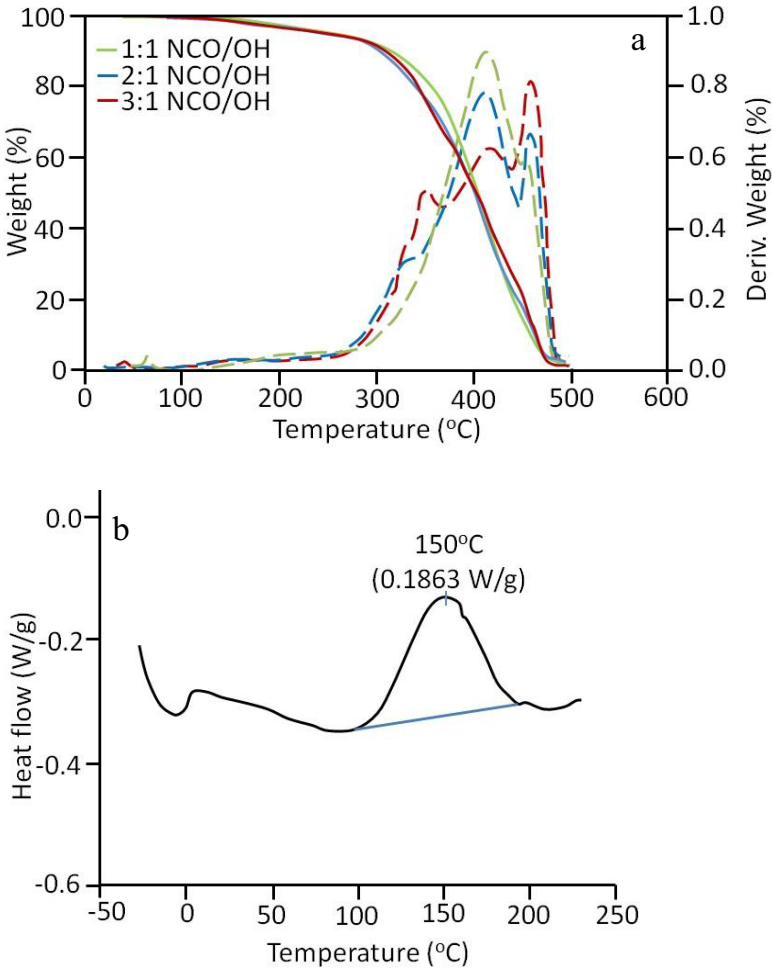

Figure 7. Thermogravimetric analyses (a) and differential scanning calorimetry thermogram (b) of corn oil-based polyurethane.

The thermal degradation of the polyurethane involves three basic mechanisms. The first refers to the dissociation of the bonds into its starting components involving the isocyanate and alcohol. The second is the breakdown of the urethane bonds with the formation of the primary amines, carbon dioxide and olefins. After which, there will be a splitting of the urethane bonds into secondary amine and carbon dioxide [17]. Figure $7 \mathrm{~b}$ also shows the DSC thermogram of the corn oil-based polyurethane at $2: 1 \mathrm{NCO} / \mathrm{OH}$ ratio with reaction time at three hours. The thermogram showed an exothermic reaction has an onset temperature of $110^{\circ} \mathrm{C}$ and peaked to a reaction temperature of $150^{\circ} \mathrm{C}$.

\section{Conclusion}

The polyurethane films were synthesized from corn oil (Zea mays L.) after the conversion of the fatty acids into a functionalized polyol with active hydroxyl groups. The variation of $\mathrm{NCO} / \mathrm{OH}$ ratio and reaction time held no significant change to the chemical composition. However, the film with $2: 1 \mathrm{NCO} / \mathrm{OH}$ ratio and three hours of reaction time showed the balance in terms of processability and physical properties.

\section{Acknowledgment}

The authors would like to express their gratitude and appreciation to Mr. Larry Reyes for his technical expertise extended in this research and the UP College of Engineering for the financial support.

\section{References}

1. K. M. Zia, H. N. Bhatti and I. A. Bhatti, React and Funct Polym 67 (2007), p. 675-692

2. H. Janik and M. Marzec, Mater Sci Eng C 48 (2015), p. $586-591$

3. K. St. John, The Spine Journal 14 (2014), p. 3038 3047

4. C. Yang, L. Fischer, S. Maranda and J. Worlitschek, Energ Buildings 87 (2015), p. 25-36

5. E. Magdaluyo, C. Aranas and J. Siglat, Philipp J Sci 140 (2011), p.25-32

6. M. Ionescu, Chemistry and Technology of Polyols for Polyurethanes (Shawbury, UK: Rapra Technology, 2005)

7. M. Aung, Z. Yaakob, S. Kamarudin and L.C. Abdullah, Ind Crop Prod 60 (2014), p.177-185

8. A. Zlatanic, C. Lava, W. Zhang and Z.S. Petrovi, J Polym Sci Pol Phys 42 (2004), p.809-819

9. S. Kabasci, Bio-Based Plastics: Materials and Applications (Pondicherry: John Wiley \& Sons, 2014)

10. S. Miao, P. Wang, Z. Su and S. Zhang, Acta Biomater 10 (2013), p.1692-1704

11. J. Clark and F. Deswarte, Introduction to Chemicals from Biomass (Chichester, West Sussex, United Kingdom: Wiley 2015)

12. Hazmi, M. Aung, L. Abdullah, M. Salleh and M. Mahmood, Ind Crop Prod 15 (2013), p.563-567

13. E. Sharmin, F. Zafar, D. Akram, M. Alam and S. Ahmad, Ind Crop Prod 76 (2015) p. 215-229

14. A. Strocchi, J Food Sci 47 (1981), p. 36-39

15. N. Hasirci and E. Aksoy, High Perform Polym 19 (2007), p.621-637

16. G. Gultekin, C. Atalay-Oral, S. Erkal, F. Sahin, D. Karastova, S.B. Tantekin-Ersolmaz and F.S. Guner, J Mater Sci - Mater M 20 (2009), p.421-431

17. J. Cauich-Rodriguez, L. Chan-Chan, F. HernandezSanchez and J. Cervantes-Uc, Degradation of Polyurethanes for Cardiovascular Applications, R. Pignatello (ed.), Advances in Biomaterials Science and Biomedical Applications (Intech, 2013), p. 51-82. 